\title{
Estudio Bibliométrico de Revistas Editadas por la UACH: Identificación de Indicadores hacia la Calidad
}

\author{
Javier Tarango Ortiz \\ Universidad Autónoma de Chihuahua, \\ Facultad de Filosofía y Letras \\ jtarango@uach.mx \\ Arturo Iván Ruiz Domínguez \\ Universidad Autónoma de Chihuahua, \\ Facultad de Filosofía y Letras \\ air_ruiz2@msn.com
}

\section{Resumen}

Es un trabajo de investigación que se refiere a un estudio bibliométrico de siete revistas editadas en la Universidad Autónoma de Chihuahua (UACH) -Lecturas Jurídicas, Excelencia Administrativa, REDICEA, Tecnociencia Chihuahua: Revista arbitrada de ciencia, tecnología y humanidades, DOXA, Metamorfosis y Synthesis-, aplicando diversos modelos métricos de información, los cuales permitieron identificar regularidades en cuanto al comportamiento de autores, concentración-dispersión, obsolescencia, vida media y envejecimiento, todo ello desde dos perspectivas: por revista y por área de conocimiento. Dado el abordaje multidisciplinario de los contenidos, la inconsistencia editorial y de periodicidad observada en las publicaciones evaluadas, se identifican diversas áreas de oportunidad hacia la mejora en cuanto a sus índices de visibilidad e impacto, así como en la determinación de criterios de estandarización en su forma de publicación.

\section{Palabras clave}

Estudios bibliométricos, publicaciones periódicas, universidades públicas mexicanas, revistas periféricas.

\section{Introducción}

Este trabajo de investigación corresponde a un estudio bibliométrico sobre publicaciones seriadas, promovidas por la Universidad Autónoma de Chihuahua (UACH), como parte de sus mecanismos de divulgación del conocimiento generado dentro de su misma estructura, a través de la colaboración de sus propios docentes e investigadores.

La importancia que cobra actualmente el análisis de las producciones editoriales de las instituciones de educación superior está en la necesidad de establecer mecanismos formales de divulgación de su propia producción y comunicación científica, apoyando a docentes e investigadores a que tengan a su alcance medios de difusión de sus productos, además de considerar otras alternativas fuera de la propia institución.

La cuestión está en que si las propias publicaciones institucionales aportan los elementos suficientes de calidad para determinarlas como canales 
viables, además de poseer elementos suficientes en cuanto a la estandarización de sus procesos de selección, que de forma eficiente, les permitan crecer como publicaciones que a corto plazo cambiando su estatus, además de que influyan en docentes e investigadores para que sus participaciones en ellas afecten positivamente en sus procesos de evaluación respecto a su producción científica.

Tales cuestiones preocupan a quienes desean participar como colaboradores de revistas propias de las instituciones de educación superior, ya que el impacto y visibilidad de las mismas suele ser tan limitado que, en la mayoría de los casos, tales contribuciones suelen pasar desapercibidas, independientemente de sus niveles de calidad, prefiriéndose usar otros canales de divulgación más efectivos.

\section{Justificación del problema}

Las universidades como entes generadores de conocimiento establecen diversos mecanismos de divulgación de sus hallazgos, los cuales se pueden agrupar en dos grandes rubros: 1). Las publicaciones externas a la misma organización (las cuales son la mayoría), y 2) aquellos mecanismos de divulgación interna (que regularmente son pocos y su comportamiento va de acuerdo con las condiciones particulares de cada institución que las genera).

Para el caso de este estudio, se observó que las publicaciones que genera la UACH caen dentro del rubro general de periféricas (esto significa que no pertenecen a la categoría de corriente principal y no están indizadas, cuestiones que se abordan a detalle a lo largo del documento), además de que presentan períodos de difusión con características irregulares, siendo los únicos proveedores de elementos para publicación, prácticamente sus mismos docentes e investigadores.

En vista de estos hechos, algunas de estas publicaciones tienen inconvenientes en solicitudes de estandarización en diversos aspectos de evaluación y presentación de contenidos, varias están tratando de obtener los registros de ISSN y no cuentan con comités editoriales estrictos que regulen la publicación. Por tanto, el presente estudio se justifica en la necesidad de diagnosticar la condición real y precisa de las publicaciones internas de la UACH a través de una descripción general del estado de tales publicaciones en sus últimos años, ya que existe la necesidad de conocer las áreas con algún tipo de inconveniente al aplicar los medidores bibliométricos.

\section{Delimitación del estudio}

El presente estudio aplica a siete publicaciones periódicas de las distintas entidades organizacionales de la UACH que las generan, tanto en modalidades impresa y electrónica, según sea el caso de la publicación. Dadas las condiciones de las publicaciones, la evaluación se desarrolla en el trascurso de los últimos años de la edición de las mismas en donde se haya observado constancia $y$ continuidad, ya que en algunas publicaciones su última época es representada por una publicación continua de 15 años.

Además, para el caso de otras publicaciones con mayor período de publicación, se cuentan con hasta 50 años en edición, pero con el inconveniente de no haberse identificado copia física o electrónica de los artículos contenidos, por lo que los sistemas de medición sólo se aplican según la característica o 
regularidad de los ejemplares obtenidos al inicio del mes de septiembre del año 2011 cuando se inicia la captura de datos.

\section{Objetivo General}

Esta investigación pretende: analizar el estado de las publicaciones periódicas editadas por la UACH, mediante la aplicación de sistemas métricos de información, además de describir el estatus actual de las últimas ediciones con regularidad en su publicación, conforme a los datos obtenidos por medio de la captura de la totalidad de artículos.

\section{Objetivos específicos}

Los objetivos específicos que pretende lograr este estudio son:

a) Clasificar por área de conocimiento, cada uno de los artículos publicados en las siete revistas generadas por la $\mathrm{UACH}, \mathrm{a}$ fin de delimitar su agrupación tomando como referencia la clasificación temática-disciplinar propuesta por el Sistema Nacional de Investigadores (SNI).

b) Conformar tablas de descriptivos generales, que incluyan: cantidad de publicaciones, años de publicación, edades máximas y cantidad de autores firmantes, elementos que permitan el desarrollo de un análisis bibliométrico específico.

c) Aplicar los principales sistemas bibliométricos que permitan determinar la condición que observan las publicaciones internas de la UACH en cuanto a su condición y derivar propuestas de elementos de mejora a través de sus indicadores de calidad.

\section{Preguntas de investigación}

Los modelos de medición existentes aplicados a los datos de la captura de artículos, permiten comprobar que:

a) ¿Las condiciones de las publicaciones editadas por la UACH permiten ser consideradas como viables a cambiar de condición a partir de la evaluación de sus indicadores bibliométricos? b) ¿Los medidores de evaluación bibliométrica se amoldan al tipo de análisis presentado?

b) ¿Los resultados que propician la derivación de las conclusiones llevan a proponer el desarrollo de modificaciones futuras en las publicaciones editadas de la UACH a fin de buscar un cambio en su condición de evaluación de su calidad?

\section{Marco Teórico}

Las universidades buscan medios de producción científica a través de sus docentes e investigadores, pretende, además, ser un medio de divulgación de la ciencia a través de sus propias publicaciones (Dávila Rodríguez y otros, 2009). Más allá de los medios de divulgación que apoyan la distribución de las revistas, se hace necesario entender la definición y clasificación que estas observan dentro de una concepción actual, entendiendo que, dentro de cualquier nivel de publicación, serán consideradas revistas científicas.

La UNESCO (2003) define a las revistas científicas publicación periódica que presenta especialmente artículos científicos, escritos por autores diferentes, e información de actualidad sobre investigación y desarrollo de cualquier área de la ciencia. Para dimensionar el nivel en que se pueden encontrar las publicaciones desarrolladas por las universidades para divulgar los hallazgos sobresalientes generados por sus 
docentes, se hace necesario entender la clasificación de las revistas de acuerdo a la propuesta integrada por López Ornelas y Cordero Arroyo (2005):

a) De primer nivel, cuando son editadas y publicadas por sociedades científicas.

b) De segundo nivel, cuando los procesos de edición, publicación y comercialización se realizan a través de grandes compañías transnacionales.

c) De tercer nivel, editadas y publicadas por entidades públicas (universidades hospitales, etc.).

Si bien la clasificación anterior se refiere al origen de la publicación, otro aspecto importante en la tipificación de recursos periódicos es de acuerdo a los tipos de contribuciones que estos reciben: a) de información (divulgan programas científicos, técnicos, educativos, etc.), b) primarias (publicación de artículos científicos originales), c) secundarias (de resúmenes) y d) terciarias (revistas de progresos científicos o tecnológicos con resúmenes de investigaciones).

Autores como Núñez Jover (2006) defiende la posibilidad de abrir un camino de valoración a aquellas prácticas científicas consideradas como ciencia periférica y por tanto, que tienen medios específicos de divulgación en revistas periféricas y naturalmente, que no están incluidas dentro del llamado mainstream (corriente principal). La asociación de estas publicaciones con países subdesarrollados y además la baja producción de modesta contribución a nivel mundial imposibilitan la tendencia hacia el reconocimiento y sobre todo al cambio de paradigma en todos los sentidos.

Como se menciona anteriormente, la defensa de las publicaciones periféricas es constante, aunque sus resultados de participación en las contribuciones a la ciencia no ayudan a una determinación hacia el reconocimiento. Es por eso que tradicionalmente la ciencia se centra en los países desarrollados, quienes además controlan y vigilan la integración de mecanismos formales de las publicaciones (propias y ajenas) convirtiendo a la producción científica en una industria con capacidad de divulgación efectiva de la ciencia, contribuciones al desarrollo de la docencia y la firme conciencia de la importancia de creación y aplicación de políticas científicas hacia la producción y comunicación científica.

Ríos Ortega (2006) prestó especial importancia al estudio de las revistas científicas periféricas ya que las considera como fundamentales en el desarrollo de la ciencia regional, además de la pretensión de cada publicación de esta naturaleza por pasare al grupo de revistas científicas. Existe una determinación de los investigadores en universidades a mantener su vigencia en el desarrollo de publicaciones en las revistas integradas dentro del grupo de corriente principal o mainstream, sin embargo, existen autores que se inclinan por fomentar que hagan sus colaboraciones además en revistas periféricas.

La imagen de una institución de educación superior o de investigación, estará sustentada en la concepción que se tenga de su producción científica, a la determinación que se tenga de establecer mecanismos de comunicación científica y el nivel al que se pretende llegar, así como a los niveles de producción de sus miembros, así como a la potencialidad para ello y disposición hacia el cambio de paradigma entre su realidad y su potencialidad en su contribución al desarrollo de la ciencia. 


\section{Análisis de resultados}

Este apartado presenta los principales hallazgos encontrados en la evaluación de las fuentes identificadas como revistas periféricas publicadas por la UACH a través de diferentes instancias (que para mayores detalles se recomienda consultar a Ruiz Domínguez, 2012), mismas que son consideradas como el universo investigativo, a través del cual se desarrolló el estudio bibliométrico. El estudio comprende los artículos publicados en siete revistas que observan un grado de formalidad y constancia en su publicación con un total de 1,175 identificados, correspondientes al período de 1990 como el más antiguo al 2011 en relación con las publicaciones de más reciente publicación. Debido a la naturaleza no regular de algunas de las publicaciones en sus procesos de edición y su acumulación, carente de un procesamiento bibliotecológico formal, de muchas de ellas no se cuenta con todos los números publicados, lo que se considera una de las limitantes de este estudio.

Los artículos publicados en las revista evaluadas, mostrados tanto en la tabla 1 como en la figura 1 , indican una distribución de los porcentaje, correspondiendo el mayor, de $42.55 \%$ del total, para la revista Synthesis, con una cantidad de 500 artículos capturados, siguiendo a esta de mayor a menor: Excelencia Administrativa con un 20.93\%, correspondientes a 246 artículos; Lecturas Jurídicas (correspondiente sólo a la última época de publicación para así ajustarla al período evaluado del resto de las revistas que componen el grupo de investigación) con 221 artículos que corresponden a un $18.80 \%$. La revista de Tecnociencia Chihuahua: Revista arbitrada de ciencia, tecnología y humanidad es observa un porcentaje de $6.55 \%$, correspondiente a 77 artículos, haciendo hincapié, que a partir del año 2007 es la revista de la UACH que ha mantenido mayor constancia en su publicación junto con la revista Synthesis. Respecto a la revista REDICEA, entra dentro de este conteo con $4.25 \%$ con 50 artículos y la revista DOXA y Metamorfosis (que son las revistas con una mayor irregularidad de publicación) tienen $4.00 \%$ y $2.89 \%$ respectivamente, donde 47 y 34 artículos publicados fueron los capturados.

Los mismos artículos publicados, antes analizados por publicación, fueron agrupados por área de la ciencia según secciona el modelo propuesto por SNI, donde el área $\mathrm{V}$ (ciencias sociales) y IV (humanidades y ciencias de la conducta) agrupan el $41.44 \% \quad y \quad 40.25 \%$ respectivamente y son los porcentajes mayores de publicación del total de las revistas (tabla 2 y figura 2). El área I (físico- matemáticas y ciencias de la tierra) sólo cuenta con cinco artículos publicados (.4255\%) el cual es el menor índice de publicación en las revistas evaluadas.

Los artículos de cada revista fueron evaluados, además, de acuerdo a los rangos de edades extremas de los años de publicación, tal como se indica en la tabla 3 , en donde la revista Lecturas Jurídicas observó que su artículo con mayor antigüedad de publicación capturado es del año 2006 y el más nuevo corresponde al año 2011, en cambio, en Tecnociencia y REDICEA son 2007 en de menor año y 2011 el de mayor año de publicación. En cuanto a la revista Synthesis, de la cual, con la inclusión de algunas revistas de la época antigua que se encontraron y capturaron, se incluyen artículos de 1990 al 2010; DOXA los años de los artículos son del 2005 al 2010, mencionando la periodicidad más irregular de la mismas ya que sólo cuenta con cuatro publicaciones en cinco años. Metamorfosis, de la misma manera que la 
revista DOXA, con irregularidad de publicación observa un período de 1998 al 2008 con cuatro números en un período de 10 años. Finalmente, y sin restar importancia, la revista Excelencia Administrativa indica que su año menor y mayor de publicación fueron 2004 y 2010. Respecto al promedio de artículos por revista evaluada calculados con base en el universo de estudio son: Lecturas Jurídicas con 13.81\%, DOXA 11.75\%, Excelencia Administrativa 11.71\%, REDICEA 10.00\%, Metamorfosis 8.50\%, Synthesis 7.46\% y Tecnociencia con $3.34 \%$, tal información es considerada en la tabla 4 y en la figura 3.

Los artículos identificados dentro del universo de investigación, de acuerdo a la materia disciplinar en la que se ubican (siguiendo nuevamente el modelo clasificatorio del SNI), indican de acuerdo a la tabla 5, una distribución siguiente: $17.61 \%$ de los artículos publicados pertenece a la materia de ciencia de educación, un 14.29\% a derecho y jurisprudencia, $13.02 \%$ a administración, 9.53\% a literatura y un $8.59 \%$ a ciencia política y administración pública, mientras que el porcentaje de materias menos publicado de la totalidad es .0851\% y corresponde por igual a las materias de arquitectura, educación física, geografía humana, geología, odontología, pesca e ingeniería textil.

El año que representa dentro de la totalidad de los datos recolectados con una mayor publicación, corresponde a 2008 con un total de 180 artículos (15.31\%), al que le sigue 2009 con 167 (14.21\%), 2010 con 158 (13.44\%) (Tabla 6), donde el año con un menor registro de captura es 1990 con 11 artículos que corresponde a .93\%. Cabe de destacar que en esta captura algunas revistas no estaban publicadas aún, mostrando un estado de en prensa.
Respecto a los artículos que incluyen lista de referencias, se contabilizaron la totalidad del $57.70 \%$ que no contaban con ella y un $42.29 \%$ que sí contaban con referencias (Tabla 7). Los artículos que fueron marcados con el indicador "NO" fue debido, como se menciona con anterioridad en el apartado de metodología, a que no se contó con el artículo fuente o por motivos de la misma naturaleza del documento, por lo cual, para estudios métricos de información con enfoque bibliométrico en aplicación de leyes métricas no es factible su utilización ya que no arrojan resultados, por tanto, para acciones posteriores de esta investigación únicamente se tomará en cuenta el porcentaje de documentos identificados que cuentan con una lista de referencia formalmente insertada dentro del mismo.

\section{Conclusiones}

Se evaluaron siete revistas editadas en diversas entidades administrativas $\mathrm{y}$ académicas de la UACH, de las cuales se observa lo siguiente:

a) Existen diferencias significativas en la constancia de las publicaciones ya que $o$ todas muestran regularidad en la constancia de sus publicaciones, lo cual inhibe a futuro el cambio de estatus en su condición de evaluación (de divulgación a científica).

b) Observan una regulación básica en la selección de artículos a publicar, considerando que sus comités editoriales no poseen los niveles de exigencia requerida por las revistas de alta calidad.

c) Aunque existen publicaciones disciplinares, otras más, que son las que manifiestan mejores elementos hacia un cambio de estatus 
editorial, poseen carácter multidisciplinario, lo que provoca que no sean distinguidas por pertenecer a procesos de divulgación de una ciencia en particular.

d) El hecho de que, prácticamente, la totalidad de las contribuciones provengan de los mismos miembros de los cuerpos docentes de la propia UACH, existe una tendencia a identificar la presencia de autores oportunistas. La élite intelectual identificada no es garantía de que sean docentes e investigadores con alta producción científica en otros medios de divulgación.

e) Se recomienda: constancia y mejora en la recepción y evaluación de los artículos a publicar, así como en la regularidad de la publicación; la elección de comités científicos de evaluación con reconocido prestigio y en la especialidad; definición de políticas editoriales institucionales para la creación de nuevas publicaciones y mejorar la estandarización de las actuales; aumentar la visibilidad de las publicaciones.

\section{Referencias}

Dávila Rodríguez, M., Guzmán Sáenz, R., Macareno Arroyo, H., Piñeres
Herrera, D., De la Rosa Barranco y Caballero-Uribe, C.V. (2009). Bibliometría: conceptos y utilidad para el estudio médico y la formación profesional. Salud Uninorte, 25, 2: 319-330.

López Ornelas, M., Cordero Arroyo, G. (2005, febrero-marzo). Un intento por definir las características generales de las revistas académicas electrónicas. Razón y Palabra, 43.

Núñez Jover, J. (2006). La ciencia y la tecnología como procesos sociales: lo que la educación científica no debería de olvidar. La Habana, Cuna: Félix Varela.

Ríos Ortega, J. (2006, enero-junio). Evaluación de normalización de las revistas contenidas en el Índice del Consejo Nacional de Ciencia y Tecnología. Investigación Bibliotecológica,20: 40, 121-148.

Ruiz Domínguez, A.I. (2012). Estudio Bibliométrico de Revistas Editadas por la UACH: Identificación de Indicadores hacia la Calidad. (Tesis de Licenciatura en Ciencias de la Información, Universidad Autónoma de Chihuahua, 2012). UNESCO, Oficina Regional de Ciencia para América Latina y el Caribe (2002). Reunión de Especialistas en Información Científica Digital. Montevideo: UNESCO. 
RECIE. Revista Electrónica Científica de Investigación Educativa

Vol. 1, núm. 1, enero-diciembre 2012, pp. 203-209. 